\title{
Lack of Evidence for Stereotypical Direction Columns in the Mouse Superior Colliculus
}

\author{
${ }^{\circledR}$ Hui Chen, ${ }^{*}$ Elise L. Savier,* Victor J. DePiero, and ${ }^{\circledR}$ Jianhua Cang \\ Department of Biology and Department of Psychology, University of Virginia, Charlottesville, Virginia 22904
}

Neurons in the visual system can be spatially organized according to their response properties such as receptive field location and feature selectivity. For example, the visual cortex of many mammalian species contains orientation and direction columns where neurons with similar preferences are clustered. Here, we examine whether such a columnar structure exists in the mouse superior colliculus (SC), a prominent visual center for motion processing. By performing large-scale physiological recording and two-photon calcium imaging in adult male and female mice, we show that direction-selective neurons in the mouse SC are not organized into stereotypical columns as a function of their preferred directions, although clusters of similarly tuned neurons are seen in a minority of mice. Nearby neurons can prefer similar or opposite directions in a largely position-independent manner. This finding holds true regardless of animal state (anesthetized vs awake, running vs stationary), SC depth (most superficial lamina vs deeper in the SC), research technique (calcium imaging vs electrophysiology), and stimulus type (drifting gratings vs moving dots, full field vs small patch). Together, these results challenge recent reports of region-specific organizations in the mouse SC and reveal how motion direction is represented in this important visual center.

Key words: columnar organization; direction selectivity; mouse visual system; silicon probes; superior colliculus; twophoton imaging

\section{Significance Statement}

The superior colliculus (SC) is an evolutionarily conserved structure that serves important functions in multimodal integration and sensorimotor transformation. Here, we determine the spatial organization of visual motion representation in the SC of mice, a popular animal model in vision research. Our results demonstrate that direction-selective neurons in the mouse SC represent stimulus directions largely independent of retinotopic location, without forming direction columns as recently reported. Our results thus provide a more nuanced and more accurate description regarding this organization and have significant implications for understanding visual processing and ethological functions of the mouse SC.

\section{Introduction}

The Superior Colliculus (SC), or its non-mammalian homolog the optic tectum, is a midbrain structure important for multimodal integration and sensorimotor transformation (May, 2006; Gandhi and Katnani, 2011). It was the most sophisticated visual center until the neocortex emerged in mammals. In mice, $>85 \%$ of retinal ganglion cells (RGCs) project to the SC (Ellis et al., 2016), making it the most prominent visual center in this species. The currently available genetic and viral tools, as well as advances

Received May 11, 2020; revised Nov. 10, 2020; accepted Nov. 12, 2020.

Author contributions: H.C., E.L.S., and J.C. designed research; H.C., E.L.S., and V.J.D. performed research; H.C., E.L.S., and V.J.D. analyzed data; H.C., E.L.S., and J.C. wrote the paper.

${ }^{*}$ H.C. and E.L.S. contributed equally to this work.

We thank Sotiris Masmanidis for providing silicon probes, Dr. Rolf Skyberg and other lab members of the Cang lab for comments on the manuscript, and the Jefferson Scholars Foundation for financial support. This work was supported by National Institutes of Health Grants EY026286 and EY020950 (to J.C.).

The authors declare no competing financial interests.

Correspondence should be addressed to Jianhua Cang at cang@virginia.edu.

https://doi.org/10.1523/JNEUROSCI.1155-20.2020

Copyright $\odot 2021$ the authors in modern imaging and recording techniques, have enabled rapid progress in functional studies of the mouse visual system. In particular, the mouse SC has received increased attention because of its clear importance in visually-guided behaviors and the rich literature of SC studies in other species (Cang et al., 2018; Ito and Feldheim, 2018).

The SC is characterized by two organizational features that are conserved over evolution, namely depth-specific segregation and topography. First, it is a layered structure with alternating strata of fibers and cell bodies. Inputs to the SC are organized according to modality along the dorso-ventral axis, with superficial layers being visual and deeper layers multimodal and premotor (Stein, 1984; May, 2006). The most superficial cellular layer of the SC is the stratum griseum superficiale (SGS), which receives direct input from $\mathrm{RGC}$ axons that enter in the stratum opticum (SO), as well as input from the visual cortex. Second, individual layers contain topographic maps of the sensory periphery or motor commands. For example, the retinal inputs are orderly mapped such that SGS neurons represent the 2D visual field along the rostro-caudal and medio-lateral axes. 
Additionally, the maps are in register across layers, allowing the SC to integrate multisensory information and to initiate orienting movements toward salient stimuli (Cang and Feldheim, 2013).

In addition to retinotopic maps, neurons in the visual system can be arranged in higher-order organizations according to their response properties, such as orientation selectivity or direction selectivity. Such maps are seen in the visual cortex of carnivores and primates, but not in rodents (Ohki et al., 2005). Intriguingly, a few recent studies reported possible region-specific organizations of feature selectivity in the mouse SC (Ahmadlou and Heimel, 2015; Feinberg and Meister, 2015; de Malmazet et al., 2018; Li et al., 2020). These reported patterns are different from the columnar organization seen in the visual cortex of higher mammals, in that not all stimulus orientations or movement directions are represented at a given retinotopic location. These findings, if confirmed, would have important implications for understanding the behavioral and ethological functions of the mouse SC. However, the reported patterns of organizations are inconsistent from each other, and are not supported by studies from our lab (Inayat et al., 2015; Savier et al., 2019).

A number of technical differences exists among these studies, such as the retinotopic location being studied, the state of the animal, visual stimuli, and the recording methods. To address these differences, we have conducted a comprehensive study to determine whether direction selectivity in the mouse SC is spatially organized. We perform both large-scale electrophysiological recording and two-photon imaging, in both anesthetized and awake mice freely running on a treadmill. We also use a large dome to deliver visual stimuli that cover almost the entire visual field, allowing us to record from a large region of the SC. Together, our data demonstrate that the preferred direction of direction-selective (DS) neurons is not systematically represented in a region-specific manner in the SC, although clusters of similarly tuned neurons are seen in a minority of animals. Our results thus provide a more nuanced and more accurate description regarding the spatial organization of the mouse SC.

\section{Materials and Methods \\ Animals}

Adult wild-type C57BL/6 mice and transgenic mice, both males and females, were used in this study. The transgenic mice were obtained by crossing Gad2-IRES-cre (The Jackson Laboratory, stock \#010802; RRID: IMSR_JAX:010802) with Ai9 (RCL-tdT, Stock \#007909; RRID: IMSR_ JAX:007909). These included 16 for two-photon calcium imaging (six wild-type and 10 GAD2xAi9), 11 for anesthetized recording, and eight for awake recording. All mice were kept on a 12/12 h light/dark cycle, with two to five animals housed per cage. All experimental procedures were approved by the University of Virginia Institutional Animal Care and Use Committee.

\section{Physiological recording in the mouse SC}

We performed large-scale physiological recordings from both anesthetized and awake mice. For anesthetized recording, we followed our published surgical procedures (Wang et al., 2009, 2010; Liu et al., 2014; Sarnaik et al., 2014; Zhao et al., 2014; Shi et al., 2017, 2018). Briefly, mice were anesthetized with an intraperitoneal injection of urethane (1.2-1.3 $\mathrm{g} / \mathrm{kg}$ in $10 \%$ saline, i.p.) and supplemented by chlorprothixene $(10 \mathrm{mg} / \mathrm{kg}$ in $4 \mathrm{mg} / \mathrm{ml}$ water, i.m.). Atropine $(0.3 \mathrm{mg} / \mathrm{kg}$ in $10 \%$ saline $)$ and dexamethasone $(2.0 \mathrm{mg} / \mathrm{kg}$ in $10 \%$ saline $)$ were administrated subcutaneously. The animal's body temperature was monitored through a rectal probe and maintained at $37^{\circ} \mathrm{C}$ through a feedback heater control module (Frederick Haer Company). Artificial tears (Henry Schein Medical) were applied to the eyes for protection during surgery. The mice were then put on a stereotaxic instrument (Narishige), with lidocaine applied on the ear bars. The scalp was shaved and skin removed to expose the skull. A metal bar was mounted on top of the skull with Metabond (Parkell). A craniotomy $\left(\sim 2.0 \times 2.0 \mathrm{~mm}^{2}\right)$ was performed on the right hemisphere for inserting the probe into the SC. The animal was then transferred to the recording station, still on heating pad, and the head bar clamped for fixation. A thin layer of silicon oil was applied on both eyes to replace the artificial tears. Additional urethane $(0.2-0.3 \mathrm{~g} / \mathrm{kg})$ was administered when necessary by monitoring toe-pinch reflex during experiments.

For awake recording, a survival surgery was first performed for head bar implant. This was done with isoflurane anesthesia ( $4 \%$ for induction, $2 \%$ for maintenance, in $\mathrm{O}_{2}, \sim 0.5 \mathrm{l} / \mathrm{min}$, VetFlo, Kent Scientific), and followed by the above procedure. After surgery, mice were given a dose of carprofen $(5 \mathrm{mg} / \mathrm{kg}$, Sub-Q) and placed in a heated chamber until ambulatory. They were then transferred back to home cage and monitored daily for pain and wound health. Two days after surgery, mice were habituated to head-fixation and running on a cylindrical treadmill for another 3-4d. Recording was performed once they were comfortable with the setup. Note that craniotomy was done on the first day of recording under isoflurane anesthesia, $\sim 3 \mathrm{~h}$ before recording.

Recordings were done using high-density multielectrode silicon microprobes developed by Sotiris Masmanidis from University of California, Los Angeles (Yang et al., 2020). The particular design we used was "128AxN Sharp," which consisted of two prongs (300- $\mu \mathrm{m}$ spacing). On each prong, 64 electrodes were aligned into three columns with $25-\mu \mathrm{m}$ vertical spacing and $20-\mu \mathrm{m}$ horizontal spacing (Fig. $1 C$ ). The probe was carefully inserted into the craniotomy and vertically through the overlaying cortex to reach the SC. The tips of probes were $\sim 2.5 \mathrm{~mm}$ below the cortical surface, at a distance of $0.2-1.5 \mathrm{~mm}$ lateral of the midline and $0.2-0.8 \mathrm{~mm}$ anterior to the $\lambda$ suture.

\section{Visual stimulation for physiological recordings}

Visual stimuli were displayed on a dome purchased from PhenoSys $\mathrm{GmbH}$. The dome was shaped as a part of a sphere that is $120 \mathrm{~cm}$ in diameter (Fig. $1 A, B$ ). It is bigger than a quarter sphere such that, with the animal at the center of the sphere, it extends from $-120^{\circ}$ to $120^{\circ}$ across azimuth $\left(0^{\circ}\right.$ representing vertical meridian $)$ and from $-30^{\circ}$ to $90^{\circ}$ in elevation $\left(0^{\circ}\right.$ representing eye level). This extent covers much of the entire visual field of a mouse, thus creating an immersive environment for visual stimulation.

Visual images were generated by our custom MATLAB scripts based on "ViRMEn" (the Virtual Reality MATLAB Engine), a freely available software package developed by Dmitriy Aronov and David Tank at Princeton University (Aronov and Tank, 2014). The images were projected by a video projector (Epson, HC1060) to a quarter-sphere panoramic mirror under the treadmill, which then reflects the images to the dome surface (Fig. 1A). In order to correct the distortion on the dome, a transformation function was generated to determines how coordinates on the $3 \mathrm{D}$ dome surface correspond to $2 \mathrm{D}$ coordinates used by the projector display on a flat screen. We did this empirically by modifying every intersection of a grid, with increasingly small spacing from $30^{\circ}, 10^{\circ}$, and $5^{\circ}$ to $2.5^{\circ}$, to straighten longitude and latitude lines on the dome surface (Fig. 1B).

Four different types of stimuli were used. First, a checkerboard was displayed over the entire dome or a smaller patch $\left(50^{\circ} \times 50^{\circ}\right.$ to $80^{\circ} \times$ $80^{\circ}$, covering the receptive fields of recorded neurons). This was used to collect local field potentials (LFPs) for estimating the depth of SC surface. Each checker was $12.5^{\circ} \times 12.5^{\circ}$ and its contrast was reversed at $1 \mathrm{~Hz}$ at $\sim 20 \mathrm{~cd} / \mathrm{m}^{2}$ mean luminance. Second, flashing spots were used to determine receptive field locations. A $10^{\circ}$ circular bright dot was flashed at different locations on a grid with $10^{\circ}$ spacing. The flashes stayed ON $\left(\sim 25 \mathrm{~cd} / \mathrm{m}^{2}\right.$ luminance) for $500 \mathrm{~ms}$ on a black background $\left(\sim 1 \mathrm{~cd} / \mathrm{m}^{2}\right.$ luminance) and OFF for $500 \mathrm{~ms}$ between stimuli. This was repeated for 10 times for each grid location in a pseudorandom sequence. We then used two different moving dots to examine direction selectivity of SC neurons. The stimuli were bright dots $\left(\sim 15 \mathrm{~cd} / \mathrm{m}^{2}\right.$ mean luminance $)$ moving on a black background, and displayed over the entire dome surface or in a smaller patch $\left(30^{\circ} \times 30^{\circ}\right.$ square covering the receptive fields of recorded neurons from one prong of the probe). The dots 

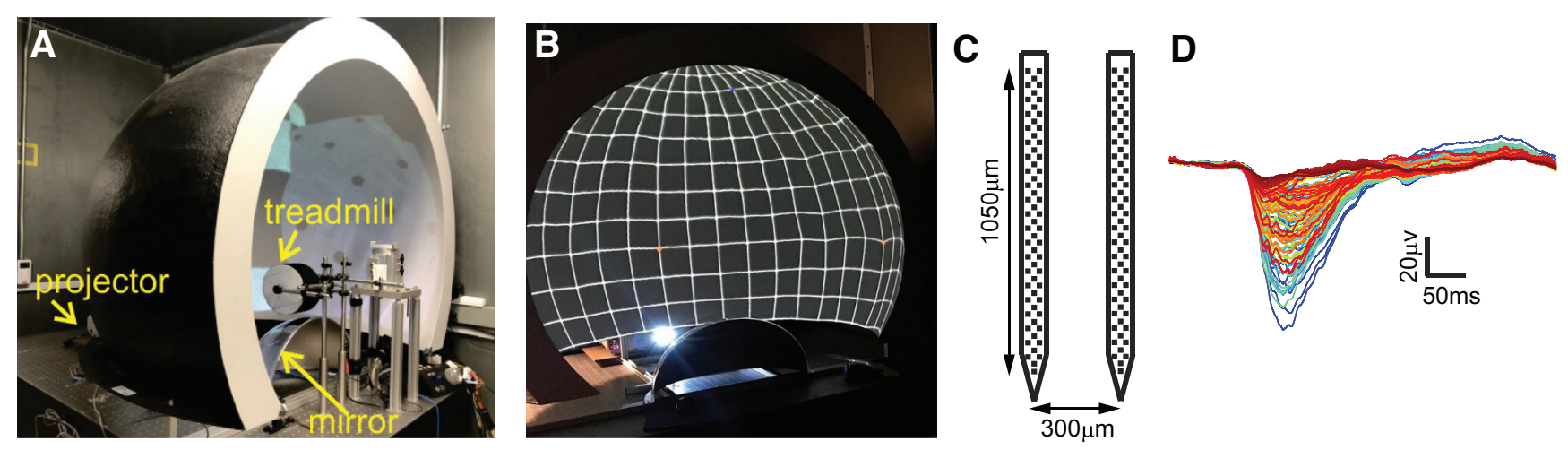

E

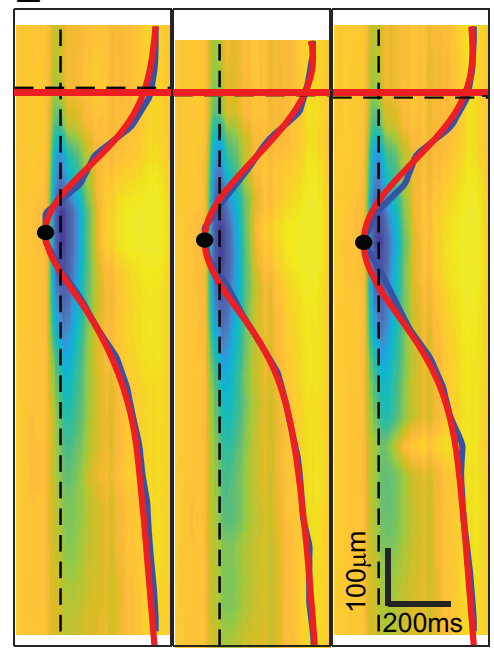

$\mathbf{F}$

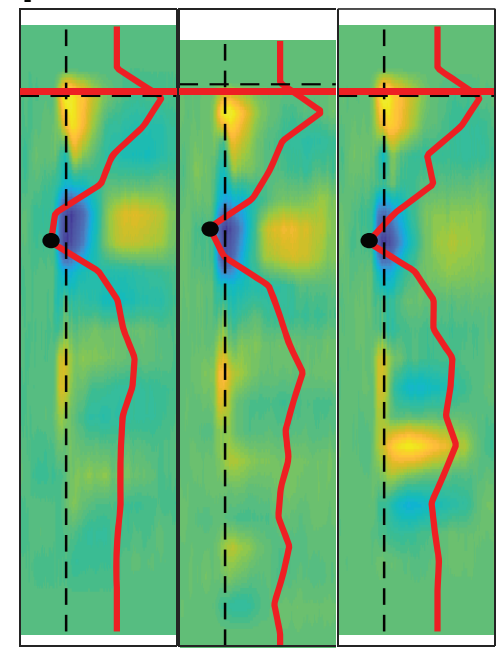

$\mathbf{G}_{0,4}$

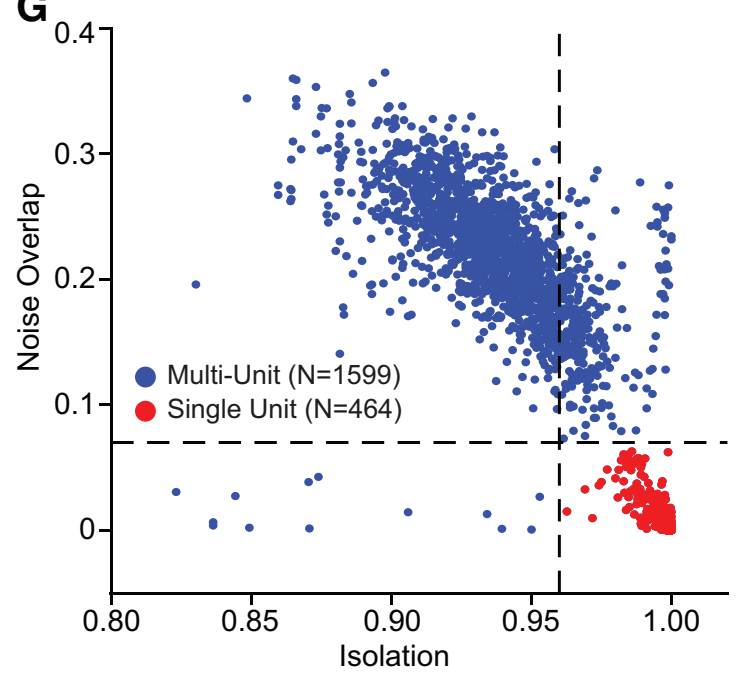

Figure 1. Physiological recording of SC neurons under a large dome. $A$, A photograph of the setup, with the video projector, panoramic mirror, and treadmill for the mouse highlighted. $\boldsymbol{B}$, Inside view of the dome surface for stimulation. A grid of longitude and latitude lines were used to adjust the display, $C$, Schematic of the two-prong silicone probes used in this study. Each dot indicates the position of one electrode contact, with a total of 64 channels per prong organized into three columns (vertical spacing: $25 \mu \mathrm{m} ;$ horizontal spacing: $20 \mu \mathrm{m})$. $\boldsymbol{D}, 0$ verlay of average LFP of all channels evoked by checkerboard reversal. $\boldsymbol{E}$, Plots of LFPs as a function of the depth ( $y$-axis) and the time ( $x$-axis). Each panel is for one column of electrodes, with cold colors indicating negative LFPs. The vertical dashed line in each panel marks the time point when the LFPs reached the maximum negative amplitude, and the LFP amplitudes at this time point of all channels is plotted as a function of depth (blue lines) and fitted to a Gaussian curve (overlaying red line). The black dashed horizontal lines in each panel and the red horizontal line indicate the estimated SC surface (for details, see Materials and Methods). $\boldsymbol{F}$, A similar plot as panel $\boldsymbol{E}$, but for CSD calculated from the LFPs to estimate SC surface. The current sink is represented in blue and the other lines mark same information as in panel $\boldsymbol{E}$. $\boldsymbol{G}$. Plot of spike sorting metrics obtained from MountainSort. Units with isolation $>0.96$ ( $x$-axis) and noise overlap $<0.07$ ( $y$ axis) were classified as single units (red dots). For details, see Materials and Methods.

( $4^{\circ}$ diameter, at a density of 450 dots of the total sphere surface area) were moving along one of eight directions $\left(0-315^{\circ}\right.$ in $45^{\circ}$ steps, speed of $30 \% \mathrm{~s}$ ) for $2 \mathrm{~s}$ and then stopped for $1 \mathrm{~s}$ before the next direction. All directions were repeated 10-12 times in a pseudorandom sequence.

\section{Data analysis for physiological recordings}

Electrical signals from the probes were recorded by a multichannel amplifier from Intan Technologies at $20 \mathrm{kHz}$. Spike and LFPs were separately filtered $(300-6000 \mathrm{~Hz}$ for spikes and $1-120 \mathrm{~Hz}$ for LFPs) and analyzed.

LFPs were used to determine the depth of SC surface (Fig. 1D-F). We compared two different approaches. First, we modified the method by Ito et al. (2017) to the following steps. (1) For each vertically aligned column of electrodes (three columns per prong), the LFPs evoked by the checkerboard reversal were averaged for each channel of electrode $(\sim 120$ repeats; Fig. $1 D)$. The LFPs were then plotted as a function of depth (Fig. $1 E, y$-axis) and time (Fig. $1 E, x$-axis). (2) We found the channel where the LFP reached the maximum negative amplitude and determined the time point when that occurred (Fig. $1 E$, black dashed vertical lines). The LFP amplitudes at this time point of all channels were plotted as a function of depth (Fig. 1E, blue curves). (3) We then fitted these data to a three-term Gaussian curve (Fig. $1 E$, red lines). (4) The position where the fitted curve had the maximum negative amplitude was determined as the center of SO. The surface was estimated to be $250 \mu \mathrm{m}$ above the SO center (Fig. 1E, black dashed horizontal lines; Zhao et al., 2014). (5) This procedure was repeated for all six columns of electrodes, and the SC surface of each prong was calculated as the mean of its three columns (Fig. 1E, red horizontal lines). Additionally, we also used current source density (CSD) analysis following our published procedures (Zhao et al., 2014). Specifically, at a given time point, the value of CSD for a particular electrode was calculated as the average difference of the LFP between four adjacent electrodes (two above and two below). We plotted CSD of each column of electrodes as a function of time. The CSD map was then smoothed by linear interpolation with a factor of 10 (Fig. 1F). The strong current sink at the beginning of the response was used to locate the SO and the surface was estimated to be $250 \mu \mathrm{m}$ above this point. We compared the estimated surface between the two methods and found the difference was only $25.6 \pm 2.8 \mu \mathrm{m}$, supporting the validity of both methods. The depth estimated from the first method (Fig. 1E)) was used in this study.

Spike waveforms were sorted offline into single-unit and multiunit spikes using a software package MountainSort (Chung et al., 2017). We separated single units and multiunits based on two metrics from MountainSort. One is noise overlap, which measures how similar the detected cluster is to randomly sampled spikes from noise. The second 

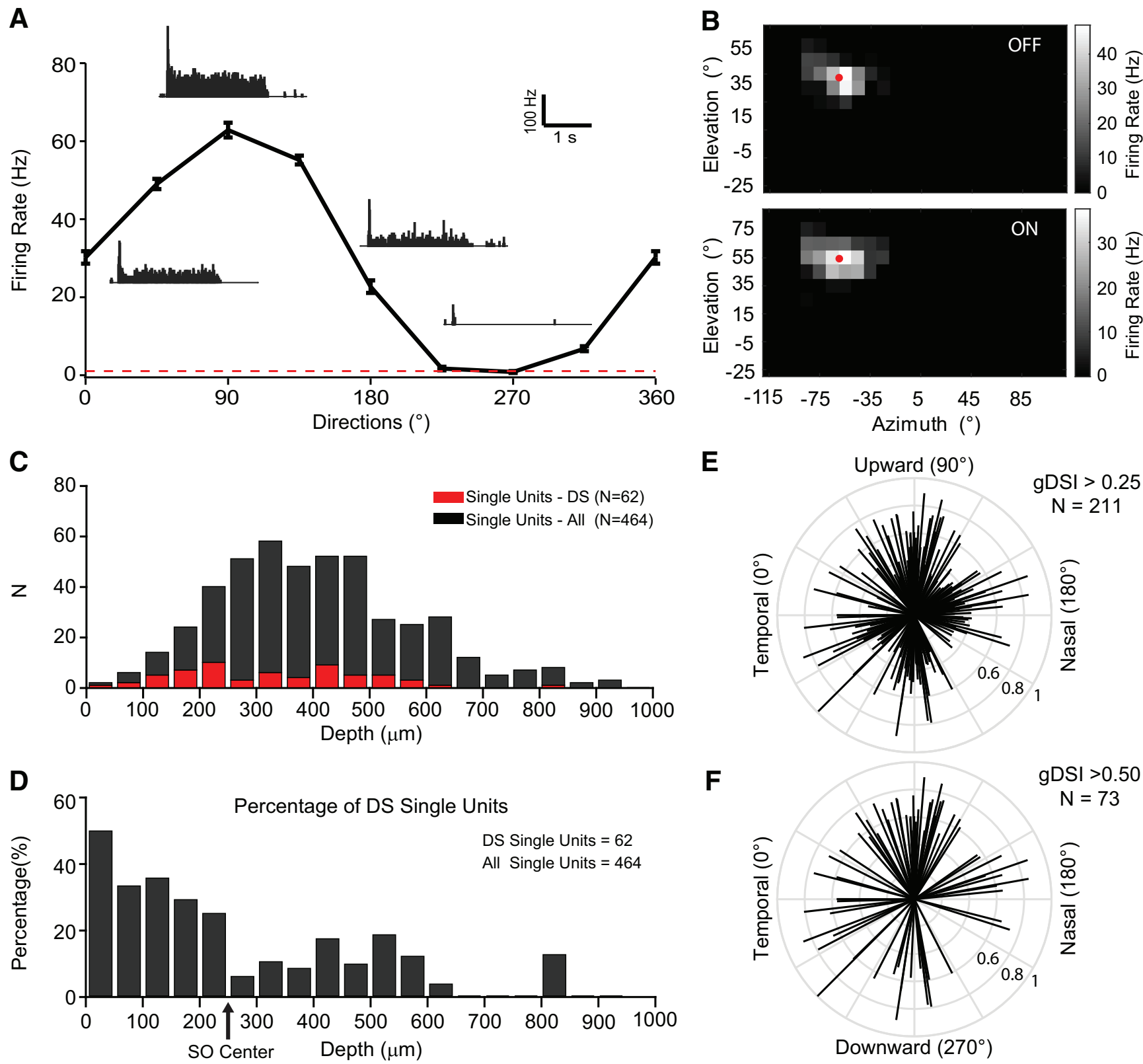

$\mathbf{F}$

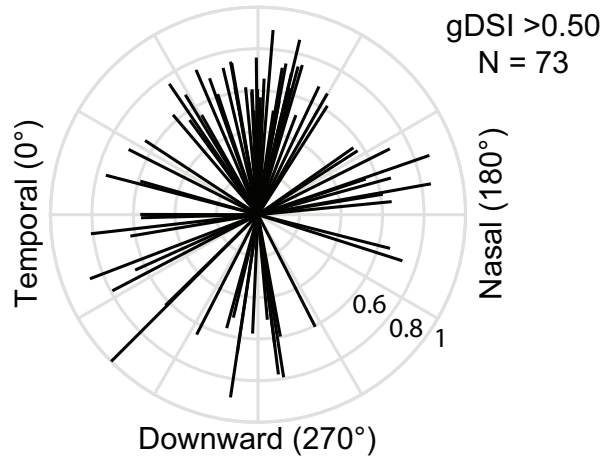

Figure 2. SC direction selectivity studied by large-scale physiological recording. $A$, An example tuning curve of a DS SC neuron (gDSI $=0.59$ ). The four insets show peristimulus time histogram at 4 directions $\left(0^{\circ}, 90^{\circ}, 180^{\circ}\right.$, and $\left.270^{\circ}\right)$. Red dashed line indicates the spontaneous rate. $\boldsymbol{B}$, Receptive field mapping plots of an example neuron, with response magnitude at each stimulus position represented in grayscale (scale bars to the right). The responses to bright spot appearance (ON) and disappearance (OFF) were separately plotted. C, Distribution of responsive single units along $S C$ depth. Red bars mark the number of DS units ( $(\mathrm{DSI}>0.25$ ). $\boldsymbol{D}$, Percentage of DS single units at each depth. $\boldsymbol{E}$, Polar plots of all DS units (gDSI $>0.25$, including 62 single units and 149 multiunits), illustrating their preferred direction (angle in the plot) and gDSI (distance from origin). $\boldsymbol{F}$, Same plot as in $\boldsymbol{E}$, for highly DS cells (gDSI $>0.50$, including 26 single units and 47 multiunits).

one is isolation, which indicates how well isolated a cluster is from other clusters. The clusters that have noise overlap $<0.07$ and isolation $>0.96$ were classified as single units (Fig. $1 G$ ). Additionally, clusters with signal-to-noise ratio $<2$ (defined as the peak absolute amplitude of the average waveform divided by the peak SD) were excluded to remove those contaminated by artifacts (Chung et al., 2017). The sorted units were subsequently analyzed to determine their responses and selectivity. First, average spike rate in response to a particular stimulus condition (e.g., movement direction for moving dots or spatial location for flashing spots) were calculated by averaging the number of spikes over the stimulus duration, across all trials and subtracting the spontaneous rate. We determined the spontaneous rate as the rate during the "blank" condition, which is a black screen for flashing spots and stationary dots for moving spots. If the evoked spiking rate was significantly different from the spontaneous responses ( $t$ test) and larger than two spikes per second, the unit was considered responsive to that condition. Units that had at least one responsive condition were included for further analysis.

For moving dots, global direction selectivity index (gDSI) and preferred direction were calculated as the vector sum of response magnitude normalized by their scalar sum (Gale and Murphy, 2014; Inayat et al., 2015; Shi et al., 2017; Barchini et al., 2018; Savier et al., 2019): $\operatorname{gDSI}=\frac{\sum R_{\theta} e^{i \theta}}{\sum R_{\theta}}$, where $R_{\theta}$ is the response magnitude at direction $\theta$. The preferred direction was the angle of the vector. For flashing spot stimulus, the grid locations in which the flashes evoked responses were determined and plotted separately for ON and OFF responses (Fig. 2B). The center of mass was calculated from these responsive locations. The 
cell's receptive field location was defined as the center of mass of the ON or OFF responses, whichever had the larger magnitude.

Finally, the animal's locomotion was monitored by a rotary encoder generating TTLs (Transistor-Transistor Logics, 100 pulses/revolution of the treadmill). The animal was considered running if the speed was higher than $1 \mathrm{~cm} / \mathrm{s}$ within the stimulus duration of a trial. To determine the effect of locomotion, we calculated a locomotion modulation index (LMI) as follow: $L M I=\frac{R_{\text {running }}-R_{\text {stationary }}}{R_{\text {running }}+R_{\text {stationary }}}$, where $R_{\text {running }}$ is the averaged response during running condition and $\mathrm{R}_{\text {stationary }}$ is the response during stationary condition (Savier et al., 2019).

Two-photon calcium imaging of SGS responses and data analysis To determine the direction preference of SGS neurons, we reanalyzed data from our recent two-photon imaging study (Savier et al., 2019), as well as performed new imaging experiments. The details of all experimental procedures, including viral injection, imaging, visual stimulation, and data analysis, were provided in the previous paper (Savier et al., 2019), with only the most relevant information listed below.

Briefly, the imaging experiments were done in the caudal pole of the SC of head-fixed mice running on a treadmill (Fig. 6A). The imaging experiments were restricted to this region because it is the only location accessible with optical methods while preserving the visual cortex. Specifically, a $\sim 2.5$-mm craniotomy was performed over $\lambda$ point in a survival surgery a few weeks before imaging. AAV1-Syn-GCaMP6f viral vector (Addgene 100837, pAAV.Syn.GCaMP6f.WPRE.SV40, titer 2 $\left.\times 10^{\wedge} 13\right)$ was injected into the SC $(50 \mathrm{nl}$ each at 200 and $400 \mu \mathrm{m}$ below the surface). After injection, a glass window that consisted of four pieces of \#1.5 coverslip glass was placed into the craniotomy. The window caught the dura and dragged it anteriorly, pushing forward the transversal sinus and exposing the SC surface for imaging. A small titanium plate was apposed to the skull to allow head-fixation during recordings.

Three to $5 \mathrm{~d}$ after the surgery, mice were habituated to head-fixation and running on the cylindrical treadmill. Imaging started $13-21 \mathrm{~d}$ postsurgery under a two-photon scanning microscope (Ultima Investigator, Bruker Nano Surface Division) with a Ti:sapphire laser (Chameleon Discovery with TPC, Coherent) at $920 \mathrm{~nm}$ using a $16 \times, 0.8$ NA Nikon objective. Imaging data were acquired using the PrairieView software (versions 5.4) with a resonant scanner at $2 \times$ optical zoom, resulting in a $412.2 \times 412.2 \mu \mathrm{m}$ field of view (at $512 \times 512$ pixel resolution). The acquisition rate was $30 \mathrm{~Hz}$, and four-frame averages were used for analysis.

Visual stimuli were displayed on an LCD monitor $(37.5 \times 30 \mathrm{~cm}$, HP L1976; and $59.7 \times 33.6 \mathrm{~cm}$, Samsung C27F398FW; $60 \mathrm{~Hz}$ refresh rate, $\sim 50 \mathrm{~cd} / \mathrm{m}^{2}$ mean luminance, $\gamma$ corrected) using MATLAB Psychophysics toolbox (Brainard, 1997; Niell and Stryker, 2008). The screen was placed $25 \mathrm{~cm}$ away from the eye contralateral to the imaging site (the right eye). The monitor was moved slightly for every imaged field of view to center the cells' receptive fields. The visual stimulus was a sinusoidal drifting grating $(100 \%$ contrast, $0.08 \mathrm{cpd}, 2 \mathrm{~Hz})$, presented on a gray background in a circular patch $\left(40^{\circ}\right.$ diameter $)$ at the center of the screen. 12 directions were used in $30^{\circ}$ steps. Each stimulus condition of the gratings was presented for $1 \mathrm{~s}$, followed by a gray screen for $3 \mathrm{~s}$, and repeated for at least 10 times in a pseudo-random manner.

We followed our published procedures to analyze the imaging data (Inayat et al., 2015; Levine et al., 2017; Barchini et al., 2018; Savier et al., 2019). Regions of interest (ROIs) were manually drawn on the average image of the collected time-series, and the intensity values of all pixels in each ROI were averaged for each frame to obtain raw $\mathrm{Ca}^{2+}$ signal. From the raw trace, $\Delta F / F_{0}=\left(F-F_{0}\right) / F_{0}$ was calculated, where $F_{0}$ was the mean of the baseline signal over six frames before stimulus onset, and $F$ was the average fluorescence signal over eight frames, one frame after stimulus onset. A cell was considered responsive if its mean $\Delta F / F_{0}$ was $>2$ SDs above its $F_{0}$ for at least one stimulus condition. The mean value of $\Delta F / F_{0}$ for each stimulus condition was then used for subsequent data analysis for all the responsive cells. To quantify the degree of direction selectivity, we calculated the vector sum gDSI $=\frac{\sum R_{\theta} e^{i \theta}}{\sum R_{\theta}}$, where $R_{\theta}$ is the response magnitude in $\Delta F / F_{0}$ at direction $\theta$. The preferred direction was the angle of the vector.

\section{Experimental design and statistical analysis}

All pooled data were presented as mean \pm SEM. Mann-Whitney test was applied for comparing data from different groups of cells, while Wilcoxon signed-rank test was applied for paired comparison. Kolmogorov-Smirnov (K-S) test was applied for comparing distributions of continuous data. All analyses and graph plotting were performed in MATLAB (MathWorks), including circular statistics by MATLAB toolbox, CircStat (Berens, 2009). The number of cells and animals, as well as details of statistical tests, are provided in Results. In figures, $* p<0.05, * * p<0.01$, and $* * * p<0.001$. No statistical methods were used to predetermine sample sizes, but our sample sizes are similar to those reported in the field. We did not randomly assign animals to groups because it is not applicable to the experimental design of this study.

\section{Results}

\section{Physiological recording of the SC in anesthetized and awake mice}

To determine whether SC neurons are organized into clusters or columns according to their preferred directions, we conducted large-scale physiological recordings. We used 128-channel silicon microprobes developed by Sotiris Masmanidis (Yang et al., 2020) to record electric signals from the SC (Fig. 1C). Spike and LFP data were separately filtered and analyzed, and LFPs were used to determine the position of SGS surface following procedures detailed in Materials and Methods (Fig. 1D-F; Zhao et al., 2014; Ito et al., 2017). The recorded spikes, both sorted single units and multiunits, were analyzed to determine their selectivity for visual stimulation (Fig. 1G). The stimuli, which were moving bright dots on a black background, were shown on a large dome that covered from $-120^{\circ}$ to $120^{\circ}$ across azimuth and from $-30^{\circ}$ to $90^{\circ}$ in elevation (Fig. $1 A, B$ ). The size of the dome allowed investigation of visual responses over a large region of the SC without the need to move visual display, thus facilitating comparison and combination of data obtained from different animals.

We recorded from both urethane-anesthetized and awake mice. In total, $n=464$ visually responsive single units were recorded from 19 mice ( $n=11$ anesthetized and 8 awake mice). As expected, the probes severely undersampled the superficial laminas, with most recorded cells at depth $>300 \mu \mathrm{m}$ below the surface, and very few from the topmost lamina (within $50 \mu \mathrm{m}$ below the surface; Fig. 2C). Overall, only 62 of these units were DS. In terms of proportion, a higher percentage in the SGS were DS than in the deeper layers (Fig. 2D), consistent with previous finding of a depth-specific distribution of direction selectivity in the SC (Inayat et al., 2015; Ito et al., 2017; Barchini et al., 2018). Within the SGS and SO (top $300 \mu \mathrm{m}$ ), 20.4\% of cells (28 out of 137 single units) were DS (gDSI >0.25), slightly lower than what was reported using single tungsten electrodes and other types of visual stimuli (Wang et al., 2010). This is likely because of the undersampling of the topmost SGS lamina, where most responsive cells are DS according to our imaging data (Inayat et al., 2015; Barchini et al., 2018; Savier et al., 2019). Finally, nearly identical depth profiles of DS units were seen in both anesthetized and awake mice $(p=0.28, \mathrm{~K}-\mathrm{S}$ stat $=0.14$ for combining multiunit and single unit, two-sample K-S test, anesthetized $n=85$, awake $n=126 ; p=0.07, \mathrm{~K}-\mathrm{S}$ stat $=0.32$ for single units only, two-sample K-S test, anesthetized $n=30$, awake $n=32$ ). In addition, no difference was observed between anesthetized and awake mice in the distribution of preferred directions $(p=0.30$, circular Kruskal-Wallis test, anesthetized $n=85$, awake $n=126$, 
combining multiunit and single unit). We therefore combined the two datasets and analyzed them together for this section.

We focused our analysis on the DS cells to determine whether their preferred directions were organized in any region-specific manner. These included the 62 single units ( 30 from anesthetized and 32 from awake) and 149 multiunits (55 from anesthetized and 94 from awake) that showed DS responses (gDSI $>0.25$ ). These units showed diverse direction preference, with a slight bias toward upward movement ( 76 units within $\pm 45^{\circ}$ of upward direction, out of 211,36\%; Fig. $2 E$ ). This bias became more obvious when analyzing highly selective units $(\mathrm{gDSI}>0.5$; $n=37 / 73,51 \%$; Fig. $2 F$ ). The preferred directions of these units appeared to form four clusters, largely along the cardinal directions (Fig. 2F).

We next analyzed the distribution of preferred directions according to retinotopic locations. The receptive fields of the DS units were determined by flashing small light spots at random locations in the dome (Fig. 2B). The preferred direction of each unit was then plotted against its receptive field location. No obvious region-specific pattern was observed for the organization of preferred direction of DS units $(n=191$ responsive units to both moving dots and flashing spots, gDSI >0.25; Fig. $3 A$ ). A diversity of tuning preferences was seen at any given retinotopic region. To quantify this, we first examined the preferred direction difference between two nearest units. The observed distribution for DS units (gDSI $>0.25$ ) was slightly different from the one randomly drawn from the dataset $(p=0.02$, two-sample $\mathrm{K}-\mathrm{S}$ test; Fig. $3 B$ ). This indicates that nearby units tended to prefer similar directions. However, this tendency was very subtle, with the difference still covering the whole range of $180^{\circ}$. Furthermore, we calculated the difference of preferred direction of pairs of DS units and plotted against the retinotopic distance between their receptive fields. The direction difference had similar mean and median values across all receptive field distances (Fig. $3 C$ ). The slightly higher density near $0^{\circ}, 90^{\circ}$, and $180^{\circ}$ direction difference likely reflected the clustering of cells' preference along cardinal directions, and it did not appear to vary with receptive field distance. This observation held true when different gDSI cutoff values were used (and the weak trend became insignificant for gDSI $>0.50$ units; Fig. $3 D-F$ ), and for both pairs combined from different animals (Fig. $3 A-G$ ) and only pairs from the same recording sessions (Fig. 3H). Finally, we grouped these DS units based on retinotopic location and plotted their preferred direction in $30^{\circ}$ bins along both azimuthal and elevation axis (Fig. 3I). Again, no systematic change was seen from this analysis. Together, these analyses demonstrate that DS cells in the SC may display a slight trend toward clustering with similarly tuned cells, but they are not organized into columns that vary systematically according to retinotopic locations.

\section{Comparison between full dome and small visual stimuli}

The above analyses were done with responses evoked by moving dots shown on the full dome. We also used moving dots in a $30^{\circ} \times 30^{\circ}$ square that covered the receptive fields of recorded neurons to determine direction selectivity. When comparing between the two stimulus conditions, we found that the preferred directions of DS neurons remained largely unchanged (Fig. 4A,B). The difference in preferred direction between the two stimuli was only $15.5 \pm 1.7^{\circ}$ for anesthetized mice (but with a consistent trend, $p=0.02, z$ statistic $=-2.27, n=89$, Wilcoxon test), and $31.8 \pm 3.1^{\circ}$ for awake mice (no consistent trend, $p=0.91, z$ statistic $=0.11, n=128$, Wilcoxon test; Fig. $4 C)$. These small differences indicate that the lack of region- specific organization is independent of stimulus size. The small visual stimuli did decrease gDSI of these units, in both anesthetized and awake mice $(p=1.4 \mathrm{e}-6, z$ statistic $=4.83$ for anesthetized; and $p=1.4 \mathrm{e}-10, z$ statistic $=6.41$, for awake, Wilcoxon test; Fig. $4 D-F$ ). To understand this reduction, we aligned all tuning curves by their preferred direction and averaged them. The peak response was similar between the two stimulus conditions under anesthesia $(p=0.22, z$ statistic $=$ -1.23 , Wilcoxon test), and showed a small but statistically significant difference in awake mice $(p=0.0096, z$ statistic $=$ -2.59 , Wilcoxon test). Importantly, the full field stimulus suppressed the tuning curves, thus increasing direction selectivity (for responses at the opposite direction, $p=1.66 \mathrm{e}-8, z$ statistic $=-5.65$ for anesthetized; and $p=1.67 \mathrm{e}-7, z$ statistic $=$ -5.23 for awake, Wilcoxon test; Fig. $4 G-H)$. Unexpectedly, we found that the peak response to moving dots was higher under anesthesia than in awake mice $(p=2.0 \mathrm{e}-5, z$ statistic $=4.27$ for full field; and $p=5.1 \mathrm{e}-5, z$ statistic $=4.0513$ for small patch, Mann-Whitney test), possibly because of an increased inhibition under awake condition as seen in V1 (Haider et al., 2013).

\section{Comparison between running and stationary behavioral states}

We next analyzed whether locomotion could affect the organization of direction preference in the SC of awake mice. For the DS cells that yielded full tuning curves under both stationary and running conditions (for details of analysis of the two states, see Materials and Methods), we analyzed changes in their preferred directions between the two states. These neurons maintained their direction preference between stationary and running conditions, whether in response to full field moving dots or a smaller patch $(p=0.60, z$ statistic $=-0.53$, Wilcoxon test, $n=42$ for full field, and $p=0.84, z$ statistic $=0.20, n=25$ for patch; Fig. $5 A$ ). This is similar to what we previously found for the superficial SGS neurons using two-photon imaging (Savier et al., 2019). In addition, locomotion did not cause systematic increase or decrease to their gDSI, although many of the cells showed a difference between the two conditions $(p=0.16, z$ statistic $=-1.39$, Wilcoxon test; $n=42$ for full field, and $p=0.22, z$ statistic $=$ $-1.22, n=25$ for patch; Fig. $5 B$ ). We then analyzed these cells' response amplitude at the preferred direction and found that they were not altered by the animal's locomotion $(p=0.22$, $z$ statistic $=1.22$, Wilcoxon test; $n=42$ for full field, and $p=0.86, z$ statistic $=0.17, n=25$ for patch; Fig. $5 C$ ), which is again similar to what we showed for the most superficial SGS neurons (Savier et al., 2019) and unlike the locomotioninduced increases in V1 (Niell and Stryker, 2010). We calculated a LMI to quantify the effect of locomotion for each unit's peak response: $L M I=\frac{R_{\text {running }}-R_{\text {stationary }}}{R_{\text {running }}+R_{\text {stationary }}}$ (Pakan et al., 2016; Savier et al., 2019). This index was close to zero and did not show any obvious trend through the depth of SC (Fig. $5 D$ ). Finally, we performed a similar analysis on the non-DS single units that were recorded in the same sessions. Like the DS cells, no prominent trend of increase or decrease was seen when comparing running and stationary conditions $(p=0.18$, $z$ statistic $=-1.35$, Wilcoxon test; $n=121$ for full field, and $p=0.42, z$ statistic $=-0.81, n=106$ for patch; Fig. $5 E)$. However, the locomotion-dependent modulation of non-DS cells appeared to be more scattered. Indeed, the LMI was more widely distributed for non-DS cells than DS cells for full field $(p=0.03, \mathrm{~K}-\mathrm{S}$ stat $=0.25$, two-sample K-S test; Fig. 5, compare 

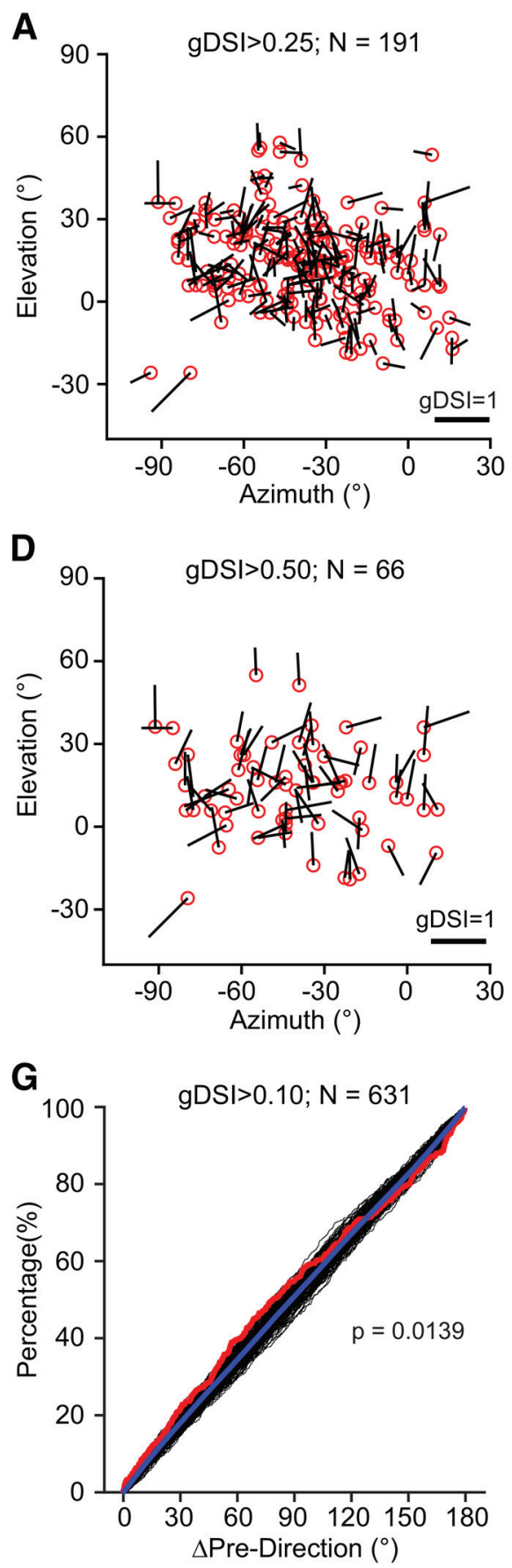
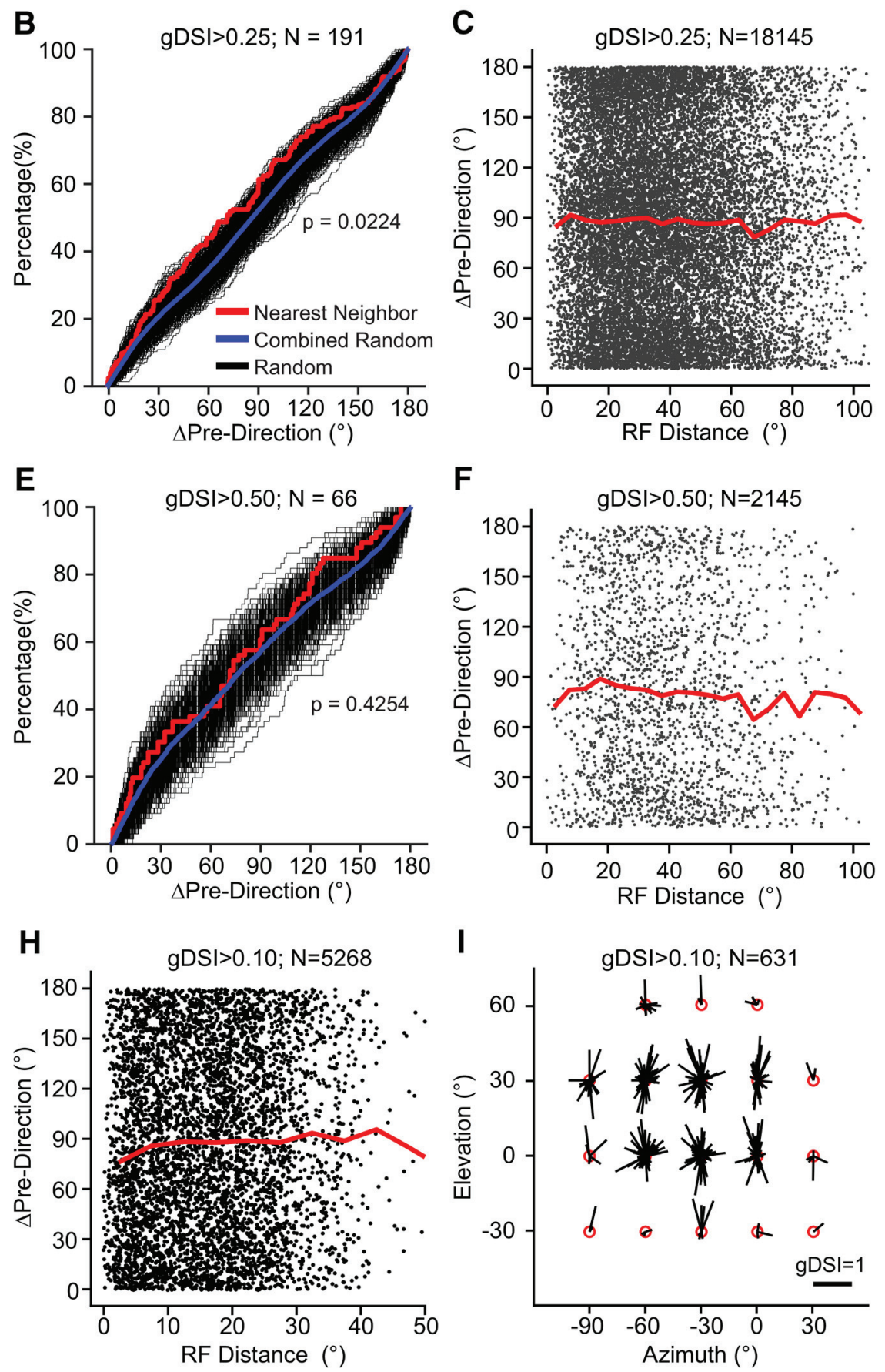

Figure 3. No region-specific organization of SC direction selectivity. $A$, Distribution of DS units as a function of their receptive fields' retinotopic location. Each red circle is one DS unit (gDSI > 0.25), and the connecting line indicate its preferred direction (angle) and gDSI (length). $\boldsymbol{B}$, Distribution of preferred direction difference between each unit and its nearest neighbor ( $g D S I>0.25$, the 191 units in $\boldsymbol{A}$, red curve). The black curves are for differences between each unit and randomly selected unit from the dataset (1000 repeats), and blue curve is the distribution combining all the randomly drawn ones. The $\mathrm{p}$ value is for comparison between the red and blue curves (K-S test). $\boldsymbol{C}$, Plot of difference in preferred direction between pairs of units (the 191 units in $\boldsymbol{A}$ ) as a function of the distance between their receptive field locations. The red line is the mean of direction difference at $5^{\circ}$ bins of receptive field distance. $\boldsymbol{D}-\boldsymbol{F}$, Same plot as $\boldsymbol{A}-$ $\boldsymbol{C}$, for units with gDSI $>0.50$. $\boldsymbol{G}$, Same plot as $\boldsymbol{B}, \boldsymbol{E}$, for units with gDSI $>0.10$. $\boldsymbol{H}$, Same plot as $\boldsymbol{C}, \boldsymbol{F}$, but only for pairs obtained in the same recordings and gDSI $>0.10$. $\boldsymbol{H}$, Distribution of DS units as a function of their receptive fields' retinotopic location, for units with gDSI $>0.10$, and the units are grouped into $30^{\circ} \times 30^{\circ}$ bins.

$D, F)$ but not for patch $(p=0.72, \mathrm{~K}-\mathrm{S}$ stat $=0.15$, two-sample $\mathrm{K}-\mathrm{S}$ test), leaving the possibility that certain subtypes of nonDS cells in the deeper SC could be specially modulated by locomotion, as suggested previously (Ito et al., 2017).

Two-photon imaging of the superficial SGS in awake mice The above experiments indicate that direction tuning in the mouse SC are not organized systematically according to retinotopic locations. However, it remained possible that some clustering may still exist in individual mice, which could "average out" when combined across animals. This possibility could not be easily tested by electrophysiological recording because of the limited DS cells that were encountered in each animal. We therefore performed two-photon calcium imaging with which many SGS neurons can be imaged simultaneously. As in our previous study (Savier et al., 2019), these imaging experiments were done 
A

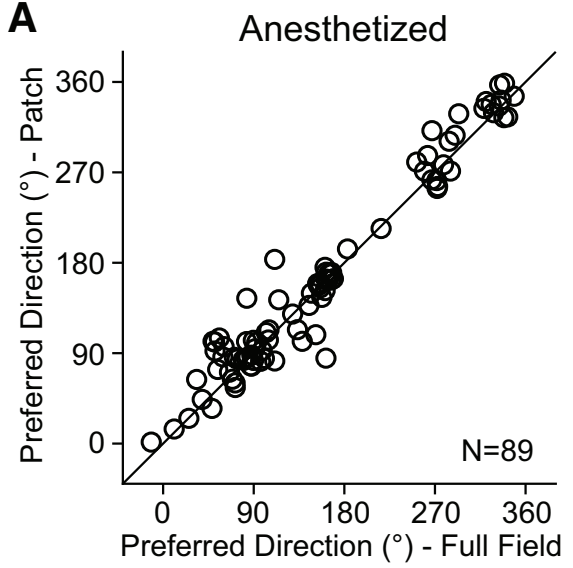

D

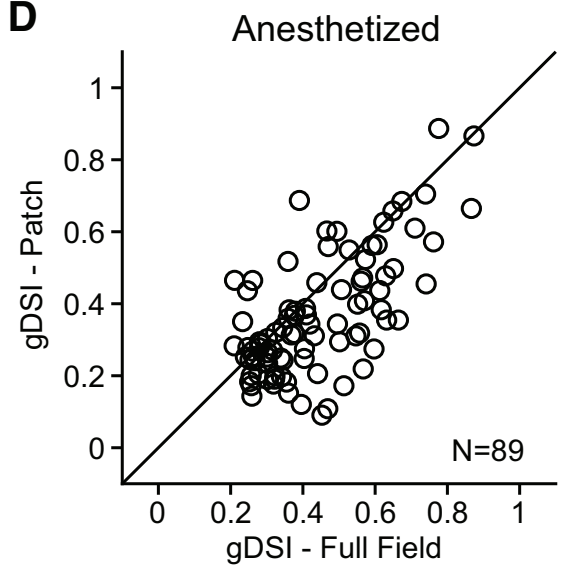

B

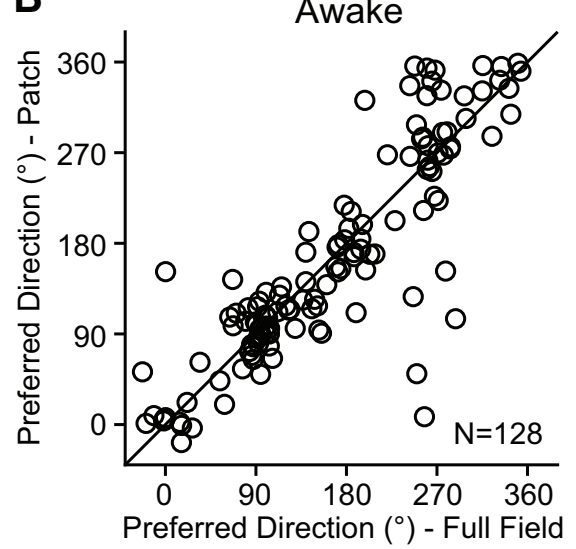

E

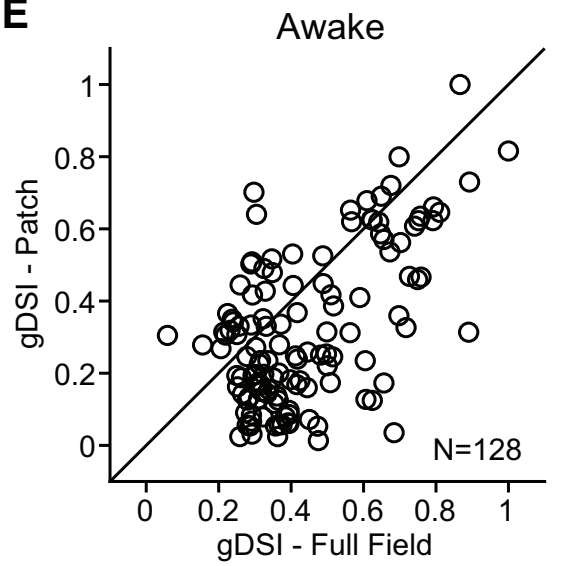

C

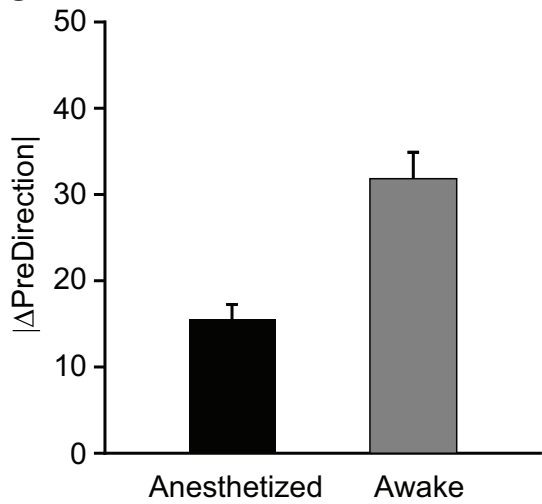

F

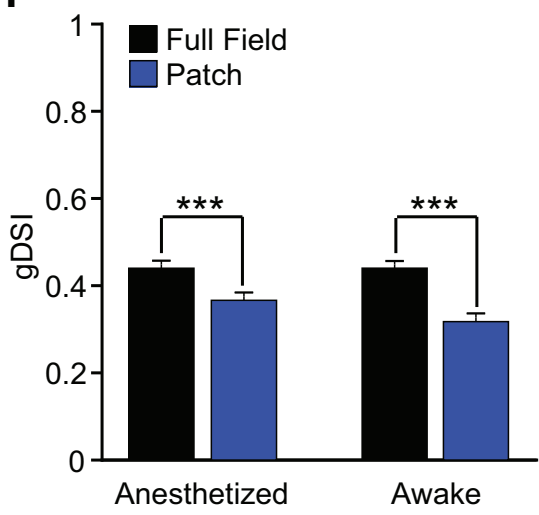

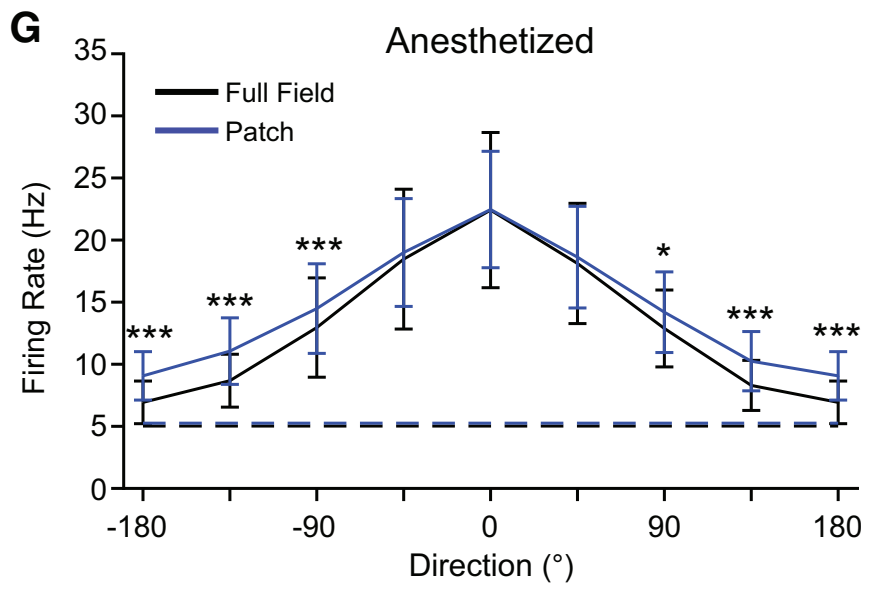

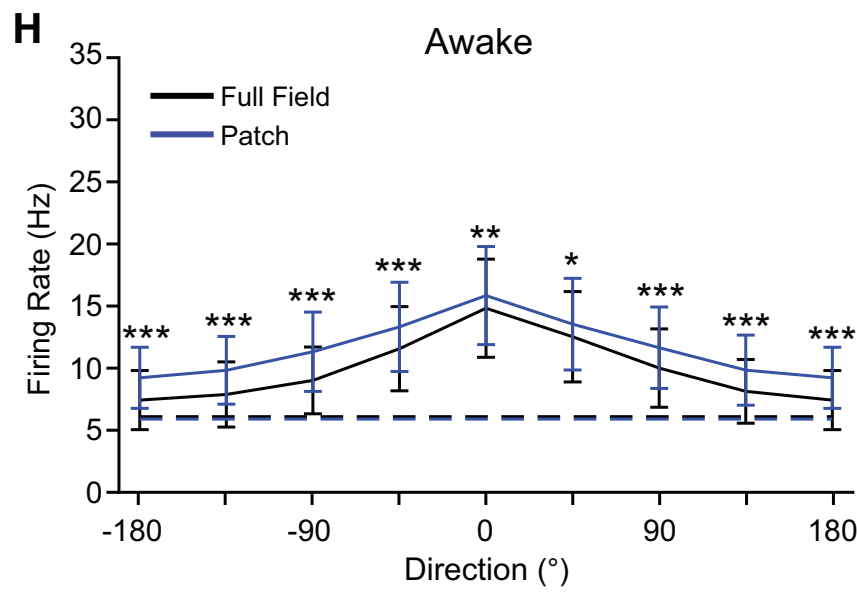

Figure 4. Stimulus size does not change preferred directions. $\boldsymbol{A}, \boldsymbol{B}$, Plots of preferred directions in response to full field ( $x$-axis) and small patch ( $y$-axis) of moving dots under anesthetized $(\boldsymbol{A})$ and awake $(\boldsymbol{B})$ conditions. $\boldsymbol{C}$, The mean difference in preferred direction between the two stimulus conditions in anesthetized and awake mice ( $p=1.82 \mathrm{e}-5, \mathrm{Mann}-\mathrm{Whitney}$ test). $\boldsymbol{D}$, $\boldsymbol{E}$, Plots of gDSI in response to full field ( $x$-axis) and small patch ( $y$-axis) of moving dots under anesthetized $(\boldsymbol{D})$ and awake $(\boldsymbol{E})$ conditions. $\boldsymbol{F}$, The difference in gDSI between the two stimulus conditions ( $p=1.4 \mathrm{e}-6$ for anesthetized; and $p=1.4 \mathrm{e}-10$ for awake, Wilcoxon test). $\mathbf{G}, \boldsymbol{H}$, Average tuning curves in response to full field (black) and small patch (blue) moving dots under anesthetized $(\boldsymbol{G})$ and awake $(\boldsymbol{H})$ conditions. The blue dashed line represents mean spontaneous firing rate. The responses to the preferred direction $\left(0^{\circ}\right)$ were similar between the two stimulus conditions, while significant differences were seen at the direction opposite to the preferred $\left( \pm 180^{\circ}\right)$. The peak response was higher under anesthesia than in awake mice $(p=2.0 \mathrm{e}-5$ comparing full field, and $p=5.1 \mathrm{e}-5$ comparing small patch, Mann-Whitney test).

at the caudal pole of the SC in awake head-fixed mice that had intact cortex (Fig. 6A). The imaged area had receptive fields in far peripheral visual space, at $90-120^{\circ}$ of azimuth $\left(0^{\circ}\right.$ representing the vertical meridian) and $30-60^{\circ}$ of elevation $\left(0^{\circ}\right.$ representing eye level). Imaging depth was limited to the topmost lamina of the SGS [within $\sim 50 \mu \mathrm{m}$ below the SC surface (sSGS)], which is enriched with DS neurons (Inayat et al., 2015; Barchini et al.,
2018; Savier et al., 2019). We re-analyzed the published data (Savier et al., 2019), as well as imaged new mice for this study. We included only mice in which at least 20 visually responsive cells were in the same field of view to analyze variability across individual animals ( $n=16$ mice, 691 cells).

In the area we imaged, which was clearly in the monocular region, many movement directions were represented (Fig. 6B). 

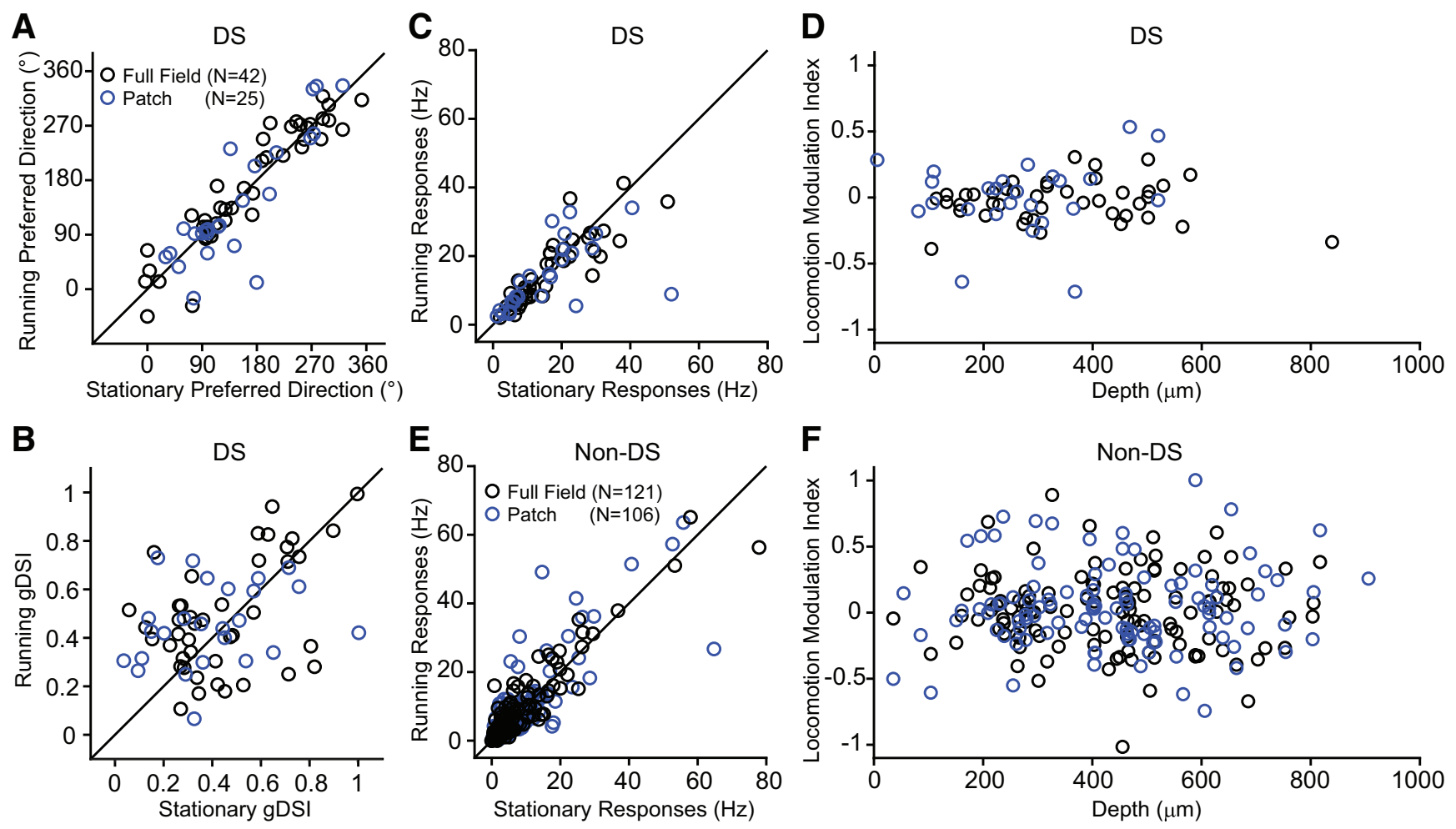

Figure 5. Comparison between running and stationary behavioral states. $A$, The preferred direction of DS neurons remained largely unchanged between stationary ( $x$-axis) and running ( $y$ axis) conditions. In this and all following plots, the responses to full field moving dots were shown by black circles, and those to a smaller patch by blue circles. The number of cells were noted in the figures. For DS group, $n=42$ for full field, and $n=25$ for patch; for non-DS group, $n=121$ for full field, and $n=106$ for patch. $\boldsymbol{B}, \boldsymbol{C}$, Comparison of gDSI (B) and peak response amplitude $(\boldsymbol{C})$ between running and stationary conditions. $\boldsymbol{D}, \mathrm{LMl}$ for the DS cells is plotted against depth in the SC. $\boldsymbol{E}, \boldsymbol{F}$, Same plots as $\boldsymbol{C}, \boldsymbol{D}$, but for non-DS neurons in the SC.

The directional preference of most sSGS neurons roughly fell into four groups, largely along the four cardinal directions (Fig. $6 B)$. This is consistent with our previous finding that direction selectivity in the SGS is inherited from the retina (Shi et al., 2017), where DS ganglion cells prefer movement in one of the four cardinal directions (Oyster and Barlow, 1967; Elstrott et al., 2008). However, this is different from a recent study that reported that $\mathrm{SC}$ cells in the monocular region almost entirely preferred temporal direction and those in the binocular region prefer nasal direction (de Malmazet et al., 2018). We did see a slight bias toward temporal compared with nasal direction ( $n=97$ with gDSI $>0.25$ and 221 with gDSI $>0.10$, within $\pm 45^{\circ}$ of temporal direction, compared with $n=77$ and 100 within $\pm 45^{\circ}$ of nasal direction), but all four cardinal directions were strongly represented (Fig. 6B).

To examine potential clustering of direction preference in individual animals, we then analyzed each mouse separately. For each field of view (Fig. $6 D-G$, left column), we plotted the preferred direction and gDSI of all visually-responsive neurons (Fig. $6 D-G$, middle column). While most fields did not show any obvious cluster (Fig. $6 D-F$ ), others did display a bias toward certain directions (Fig. 6G). Because a cell's preferred direction and gDSI was represented as a vector, we calculated a sum of all the vectors for each field of view (normalized by the scalar sum) to represent the extent of the clustering (Fig. 6C). This vector sum, which we call region selectivity index (RSI), was quite variable among the imaged mice, ranging from 0.05 to 0.79 (mean: $0.32 \pm 0.05$ ), with the examples in Figure $6 D-G$ representing 25 th, 50th, 75th, and 100th percentiles of the data set (\#4, \#8, \#12, and \#16, rank ordered by the RSI, from low to high). Furthermore, we compared the preferred direction of pairs of DS neurons (gDSI $>0.10$ ). Over all pairs of DS neurons for each individual, the difference in direction preference distributed over the entire range from $0-180^{\circ}$ (Fig. $6 D-G$, right column), with bias toward $0^{\circ}$ (i.e., similar direction preference) only in individuals with the highest RSI values. Importantly, direction difference did not show a clear trend according to the distance between pairs. Neurons that were close to each other (e.g., $<50 \mu \mathrm{m}$ ) could prefer similar or opposite directions, just as those cells that were further apart (e.g., $\sim 200 \mu \mathrm{m}$ ), confirming what we showed previously in anesthetized mice with cortex removed (Inayat et al., 2015). Because all mice were imaged at similar locations (i.e., the medial-caudal sSGS), as constrained by the surgical procedure and confirmed by receptive field position, these data demonstrate intriguing variabilities among individual animals.

Finally, to examine whether the individual variability could be because of unstable tuning of DS neurons, we repeatedly imaged the same fields of view and tracked the response properties of individual ROIs. Only ROIs that could be reliably identified across sessions were used for this analysis. Overall, we were able to track 107 neurons in seven mice for up to six weeks (Fig. 7). The vast majority of these cells showed stable tuning for stimulus direction (Fig. $7 B, C$ ), with $57 \%$ of cells displaying fewer than $10^{\circ}$ difference between successive recording sessions (Fig. $7 D$, median: $7.7^{\circ}$; mean: $16.7 \pm 1.4^{\circ}, n=373$ comparisons between successive sessions, 107 neurons from seven mice). The neurons with large changes were mostly those with low gDSI (Fig. 7E, $r=-0.3, p=4.15 \times 10^{-9}, n=373$ ). In other words, our chronic imaging experiments demonstrate that DS neurons in the sSGS maintain stable direction tuning, with the direction preference clusters seen in some animals consistent across recording sessions (Fig. 7A, the blue clusters). 

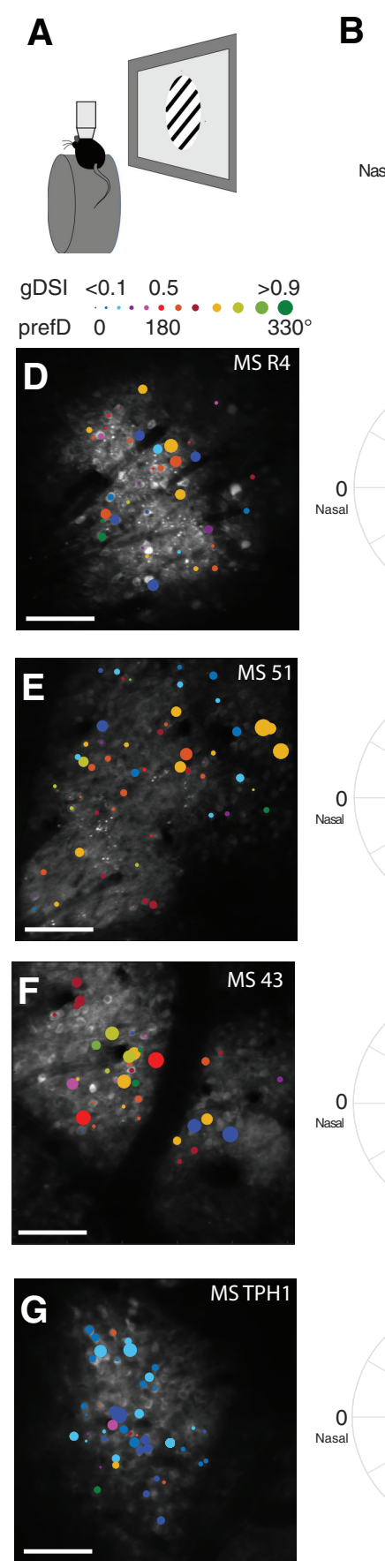
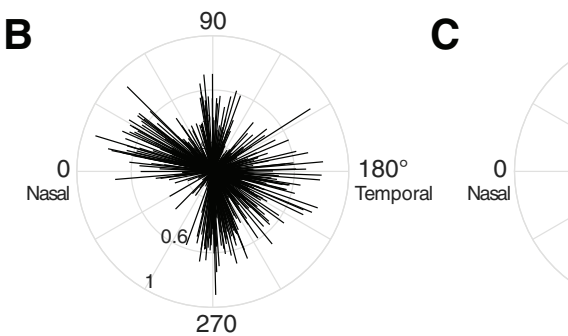

90
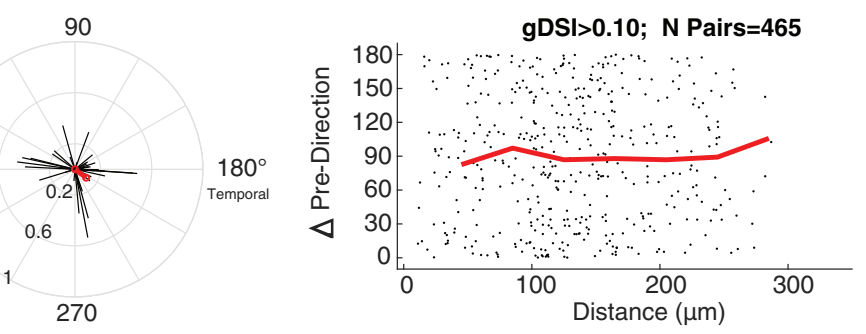

90
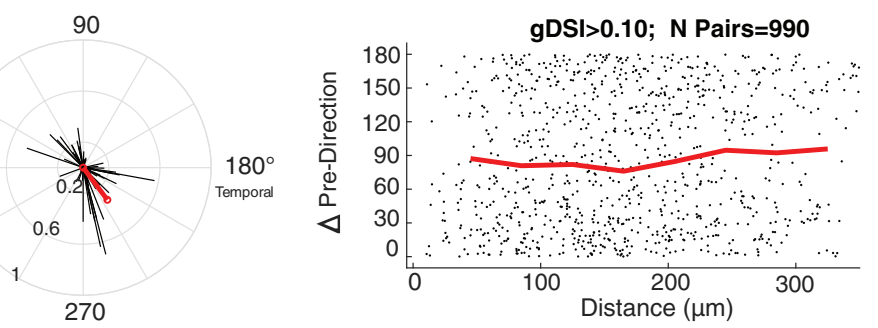

90

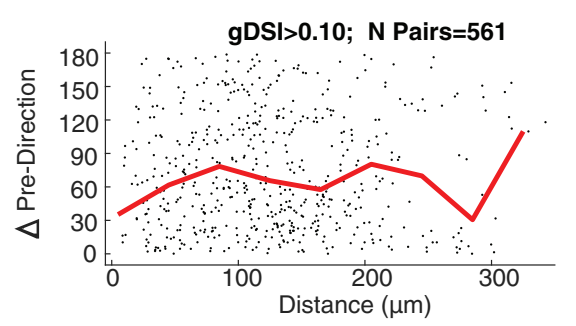

90
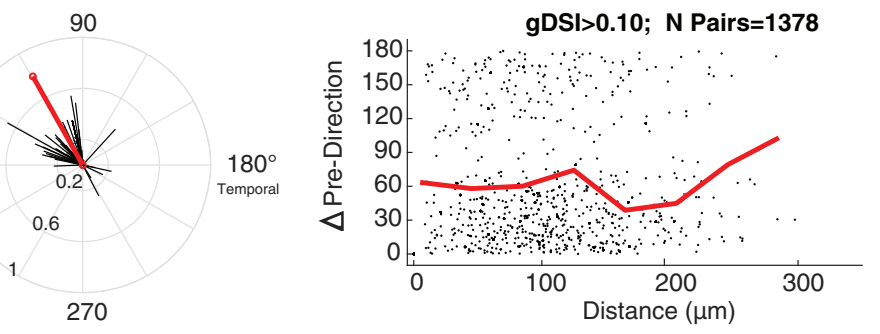

Figure 6. Characterization of the distribution of preferred directions in the superficial SGS using two-photon calcium imaging. $\boldsymbol{A}$, Experimental setup. Stimulus was presented on a movable screen, $25 \mathrm{~cm}$ away from the mouse's eye and centered on the retinotopic location. $\boldsymbol{B}$, Vector representation of the preferred direction (direction) and gDSI (magnitude) of all responsive neurons ( $n=691$ from 16 mice). C, Distribution of RSI (region selectivity index). Each line represents an RSI, which is the vector sum of all visually responsive cells in that field of view. $\mathbf{D}-\mathbf{G}$, Four individual examples, representing 25th $(\boldsymbol{D}), 50$ th $(\boldsymbol{E}), 75$ th $(\boldsymbol{F})$, and 100th $(\boldsymbol{G})$ percentiles of the dataset (\#4, \#8. \#12, and \#16, of 16 mice rank ordered from low to high by the vector sum in panel C). The left column shows the fields of view, with colored circles representing the cell's preferred direction (color) and gDSI (size of the circles). Scale bar: $100 \mu \mathrm{m}$. The polar plots in the middle show the direction tuning of responsive cells (black). The red lines are the vector sum of all cells and correspond to the red lines in panel $\boldsymbol{C}$. The plots on the right show the difference in preferred direction $\left(\Delta^{\circ}\right)$ as a function of distance $(\mu \mathrm{m})$ between pairs of ROls with gDSI $>0.1$. Average of $10-\mu \mathrm{m}$ bins is represented in red. prefD, preferred direction; gDSI, global selectivity index.

\section{Discussion}

\section{Spatial organization of visual motion representation in the} mouse SC

In this study, we have determined the spatial organization of visual motion representation in the mouse SC. Our results demonstrate that DS neurons in the SC are not organized into stereotypical columns according to their preferred directions. A minority of animals did show some clustering of neurons with similarly tuning, but nearby neurons can overall prefer similar or opposite directions that is largely independent of retinotopic position and proximity. This finding holds true under both anesthetized and awake conditions, for superficial and deeper laminae, regardless of stimulus size, and independent of animal's locomotion state. Together, these results provide a more nuanced and more accurate description of collicular organization than recent reports of this issue and thus have significant implications 

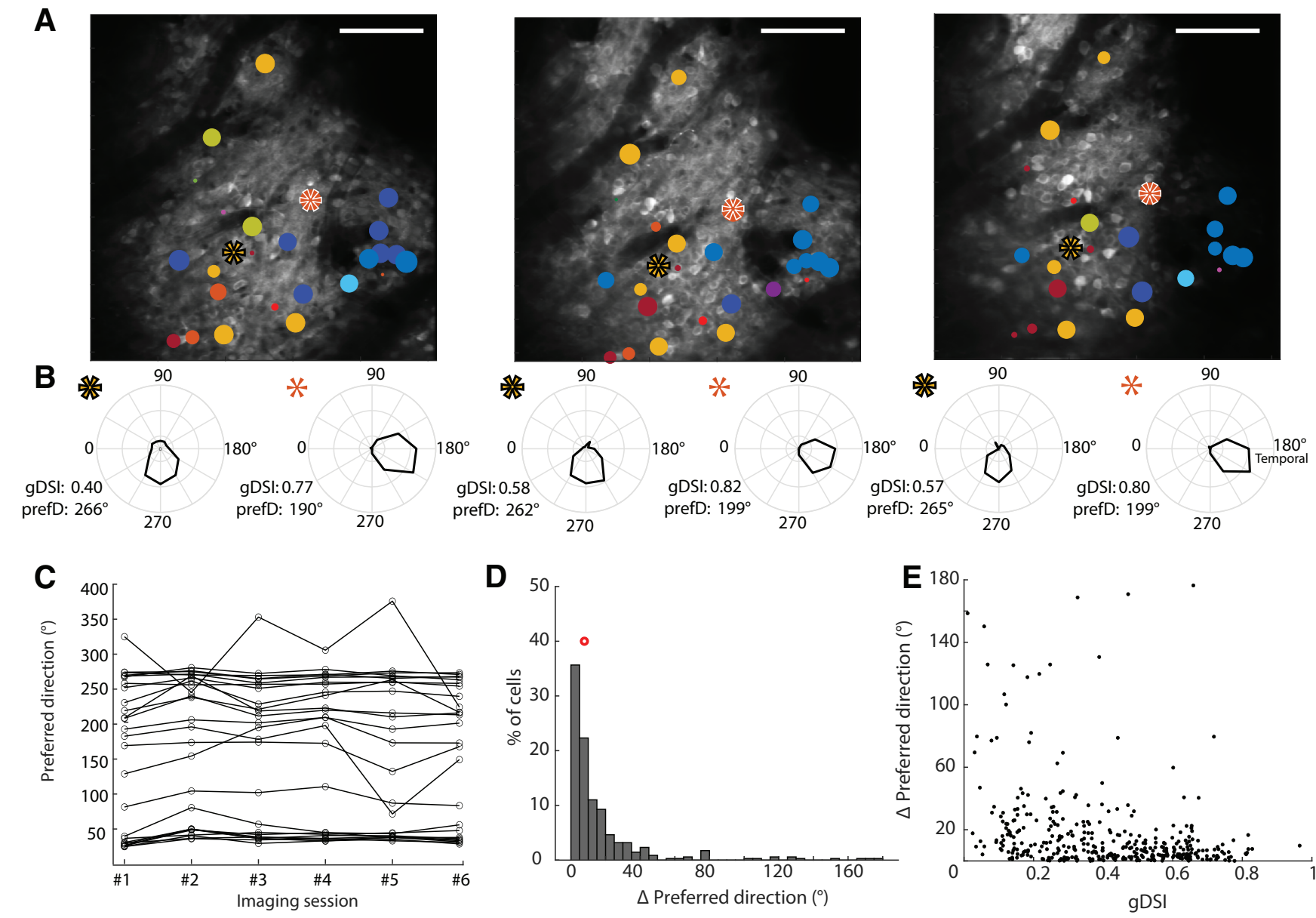

Figure 7. Stability of superficial SGS direction selectivity revealed by chronic imaging. $\boldsymbol{A}$, An example field of view imaged on three different days over approximately four weeks. Only the ROls that can be reliably identified are shown according to the same color scale in Figure 6 . Scale bar: 100um. $\boldsymbol{B}$, Tuning curves of two example neurons at the three imaging sessions marked with a star at the corresponding visual field location. C, Preferred direction as a function of recording sessions of 26 neurons from this mouse. $\boldsymbol{D}$, Histogram of the difference in preferred direction between successive imaging sessions (the red circle marks the median, $7.8^{\circ}, n=373$ comparisons, 107 neurons from seven animals, each measured two to six times). $\boldsymbol{E}$, The difference in cell's preferred direction between successive sessions as a function of its gDSI in the first of two sessions $\left(r=-0.3, p=4.15 \times 10^{-9}, n=373\right)$.

for understanding visual processing and ethological functions of the mouse SC.

The debate regarding spatial organization of SC feature selectivity dates back to early days of vision research and goes beyond mice. In studying the cat SC, McIlwain and Buser noted that when DS units were recorded, their preferred directions tended to be the same as those of their immediate neighbors (McIlwain and Buser, 1968). Similarly in ground squirrel, Michael found that successively recorded units in vertical penetrations of the SC preferred same directions (Michael, 1972). Furthermore, it was shown that DS cells in the cat SC are retinotopically organized such that they signaled centrifugal movements (Straschill and Hoffmann, 1969). However, the experiments by Sterling and Wickelgren did not support the existence of direction columns in the cat SC, although they did report that the vast majority of the DS cells they recorded preferred horizontal movements (Sterling and Wickelgren, 1969). These discrepancies and observations, as far as we know, have not been resolved or confirmed.

Dräger and Hubel were the first to study receptive field properties of mouse SC neurons, including direction selectivity (Dräger and Hubel, 1975a,b, 1976). They found that the vast majority of the recorded DS neurons (35 of 38 cells) preferred upward motion (Dräger and Hubel, 1975b). A slight bias toward upward motion is indeed seen in our physiology data, but nowhere near that extent. More importantly, all directions, especially the four cardinal directions, were represented in the mouse SC. However, it was suggested that the mouse SC might represent motion direction in a location-specific manner. For example, it was reported that "a sharp transition" in direction preference existed at the monocular-binocular border in the mouse SC, such that DS neurons in the binocular zone (central visual field) overwhelmingly preferred nasal movements, while those in the monocular zone (peripheral) preferred temporal ones (de Malmazet et al., 2018). Curiously, very few cells were shown to prefer upward or downward movements in that study. A more recent study also reported "locally biased representation" of motion direction, which contains large patches of the SC that prefer similar directions $\left(\sim 30^{\circ}\right.$ in visual angle, and up to $\sim 350 \mu \mathrm{m}$ in depth; Li et al., 2020). However, the patterns revealed by the two studies are inconsistent, and are not supported by our experimental studies and data analysis. The reason for these discrepancies remains unknown, but could be because of the individual differences we revealed here. Some mice did show clusters where nearby neurons preferred similar directions, but they were a minority. These individual differences should be taken into consideration in future studies, such as using individual animals as a unit of analysis and regularly refreshing mouse lines including even the wild type.

A columnar organization in the mouse SC has also been reported for the representation of orientation, but the exact patterns were again inconsistent between the two studies 
(Ahmadlou and Heimel, 2015; Feinberg and Meister, 2015). We did not examine orientation selectivity because very few cells in the most superficial lamina were orientation selective and only moving dots were used in physiology recordings. However, our data of direction selectivity are not congruent with the existence of orientation columns, because of the orthogonal relationship between orientation and direction preference (Li et al., 2020; but see de Malmazet et al., 2018). Nevertheless, it is tantalizing to ponder why so many studies, in several species, have observed clusters or columns of DS/OS neurons in the SC. It remains possible that the SC may indeed encode visual features in a regionspecific manner, but beyond direction or orientation selectivity in a way that we still do not understand. Future studies with more sophisticated stimulus design and behavioral paradigms may help resolve this interesting puzzle.

\section{Locomotion-dependent modulation of SC responses}

In this study, we also examined locomotion-dependent modulation of SC responses. This was motivated by the series of studies showing that V1 neurons dramatically increase their visuallyevoked responses when the mouse is running (Niell and Stryker, 2010; Busse et al., 2017). We had previously shown that the most superficial SGS neurons did not display such a profound potentiation (Savier et al., 2019). Instead, only a minority of neurons displayed significant changes during locomotion, which included both increase and decrease in response amplitude. Here, we expanded this finding to deeper and non-DS SC neurons. Again, both response increase and decrease were seen, but the level of modulation was more widespread. The observed heterogeneity is consistent with a previous study of locomotion-dependent modulation in the mouse SC (Ito et al., 2017). Nevertheless, we were surprised by the lack of overwhelming potentiation during locomotion, given that cortical input is known to increase SGS response gain (Zhao et al., 2014). This is likely because of the fact that V1 layer 5 neurons, where cortico-collicular projections originate, only display weak potentiation during locomotion, with an amplitude much smaller than layer $2 / 3$ neurons (Hoy and Niell, 2015; Dadarlat and Stryker, 2017). Finally, our data leave open the possibility that certain cell types in the SC could still be specifically modulated by locomotion. For example, widefield vertical (WFV) cells send outputs to the lateral posterior nucleus (the rodent pulvinar), which has been shown to carry locomotion signal to V1 (Roth et al., 2016). Future studies with cell type precision will be needed to determine how WFV and other neuron types are modulated by locomotion.

\section{References}

Ahmadlou M, Heimel JA (2015) Preference for concentric orientations in the mouse superior colliculus. Nat Commun 6:6773.

Aronov D, Tank DW (2014) Engagement of neural circuits underlying 2D spatial navigation in a rodent virtual reality system. Neuron 84:442-456.

Barchini J, Shi X, Chen H, Cang J (2018) Bidirectional encoding of motion contrast in the mouse superior colliculus. Elife 7:e35261.

Berens P (2009) CircStat: a MATLAB toolbox for circular statistics. J Stat Soft 31.

Brainard DH (1997) The psychophysics toolbox. Spat Vis 10:433-436.

Busse L, Cardin JA, Chiappe ME, Halassa MM, McGinley MJ, Yamashita T, Saleem AB (2017) Sensation during active behaviors. J Neurosci 37:10826-10834.

Cang J, Feldheim DA (2013) Developmental mechanisms of topographic map formation and alignment. Annu Rev Neurosci 36:51-77.

Cang J, Savier E, Barchini J, Liu X (2018) Visual function, organization, and development of the mouse superior colliculus. Annu Rev Vis Sci 4:239262.
Chung JE, Magland JF, Barnett AH, Tolosa VM, Tooker AC, Lee KY, Shah KG, Felix SH, Frank LM, Greengard LF (2017) A fully automated approach to spike sorting. Neuron 95:1381-1394.e6.

Dadarlat MC, Stryker MP (2017) Locomotion enhances neural encoding of visual stimuli in mouse V1. J Neurosci 37:3764-3775.

de Malmazet D, Kühn NK, Farrow K (2018) Retinotopic separation of nasal and temporal motion selectivity in the mouse superior colliculus. Curr Biol 28:2961-2969.e4.

Dräger UC, Hubel DH (1975a) Physiology of visual cells in mouse superior colliculus and correlation with somatosensory and auditory input. Nature 253:203-204.

Dräger UC, Hubel DH (1975b) Responses to visual stimulation and relationship between visual, auditory, and somatosensory inputs in mouse superior colliculus. J Neurophysiol 38:690-713.

Dräger UC, Hubel DH (1976) Topography of visual and somatosensory projections to mouse superior colliculus. J Neurophysiol 39:91-101.

Ellis EM, Gauvain G, Sivyer B, Murphy GJ (2016) Shared and distinct retinal input to the mouse superior colliculus and dorsal lateral geniculate nucleus. J Neurophysiol 116:602-610.

Elstrott J, Anishchenko A, Greschner M, Sher A, Litke AM, Chichilnisky EJ, Feller MB (2008) Direction selectivity in the retina is established independent of visual experience and cholinergic retinal waves. Neuron 58:499-506.

Feinberg EH, Meister M (2015) Orientation columns in the mouse superior colliculus. Nature 519:229-232.

Gale SD, Murphy GJ (2014) Distinct representation and distribution of visual information by specific cell types in mouse superficial superior colliculus. J Neurosci 34:13458-13471.

Gandhi NJ, Katnani HA (2011) Motor functions of the superior colliculus. Annu Rev Neurosci 34:205-231.

Haider B, Häusser M, Carandini M (2013) Inhibition dominates sensory responses in the awake cortex. Nature 493:97-100.

Hoy JL, Niell CM (2015) Layer-specific refinement of visual cortex function after eye opening in the awake mouse. J Neurosci 35:3370-3383.

Inayat S, Barchini J, Chen H, Feng L, Liu X, Cang J (2015) Neurons in the most superficial lamina of the mouse superior colliculus are highly selective for stimulus direction. J Neurosci 35:7992-8003.

Ito S, Feldheim DA (2018) The mouse superior colliculus: an emerging model for studying circuit formation and function. Front Neural Circuits 12:10.

Ito S, Feldheim DA, Litke AM (2017) Segregation of visual response properties in the mouse superior colliculus and their modulation during locomotion. J Neurosci 37:8428-8443.

Levine JN, Chen H, Gu Y, Cang J (2017) Environmental enrichment rescues binocular matching of orientation preference in the mouse visual cortex. J Neurosci 37:5822-5833.

Li YT, Turan Z, Meister M (2020) Functional architecture of motion direction in the mouse superior colliculus. Curr Biol 30:3304-3315.e4.

Liu M, Wang L, Cang J (2014) Different roles of axon guidance cues and patterned spontaneous activity in establishing receptive fields in the mouse superior colliculus. Front Neural Circuits 8:23.

May PJ (2006) The mammalian superior colliculus: laminar structure and connections. Prog Brain Res 151:321-378.

McIlwain JT, Buser P (1968) Receptive fields of single cells in the cat's superior colliculus. Exp Brain Res 5:314-325.

Michael CR (1972) Functional organization of cells in superior colliculus of the ground squirrel. J Neurophysiol 35:833-846.

Niell CM, Stryker MP (2008) Highly selective receptive fields in mouse visual cortex. J Neurosci 28:7520-7536.

Niell CM, Stryker MP (2010) Modulation of visual responses by behavioral state in mouse visual cortex. Neuron 65:472-479.

Ohki K, Chung S, Ch'ng YH, Kara P, Reid RC (2005) Functional imaging with cellular resolution reveals precise micro-architecture in visual cortex. Nature 433:597-603.

Oyster CW, Barlow HB (1967) Direction-selective units in rabbit retina: distribution of preferred directions. Science 155:841-842.

Pakan JM, Lowe SC, Dylda E, Keemink SW, Currie SP, Coutts CA, Rochefort NL (2016) Behavioral-state modulation of inhibition is context-dependent and cell type specific in mouse visual cortex. Elife 5

Roth MM, Dahmen JC, Muir DR, Imhof F, Martini FJ, Hofer SB (2016) Thalamic nuclei convey diverse contextual information to layer 1 of visual cortex. Nat Neurosci 19:299-307. 
Sarnaik R, Chen H, Liu X, Cang J (2014) Genetic disruption of the on visual pathway affects cortical orientation selectivity and contrast sensitivity in mice. J Neurophysiol 111:2276-2286.

Savier EL, Chen H, Cang J (2019) Effects of locomotion on visual responses in the mouse superior colliculus. J Neurosci 39:9360-9368.

Shi X, Barchini J, Ledesma HA, Koren D, Jin Y, Liu X, Wei W, Cang J (2017) Retinal origin of direction selectivity in the superior colliculus. Nat Neurosci 20:550-558.

Shi X, Jin Y, Cang J (2018) Transformation of feature selectivity from membrane potential to spikes in the mouse superior colliculus. Front Cell Neurosci 12:163.

Stein BE (1984) Development of the superior colliculus. Annu Rev Neurosci 7:95-125.

Sterling P, Wickelgren BG (1969) Visual receptive fields in the superior colliculus of the cat. J Neurophysiol 32:1-15.
Straschill M, Hoffmann KP (1969) Response characteristics of movementdetecting neurons in pretectal region of the cat. Exp Neurol 25:165-176.

Wang L, Rangarajan KV, Lawhn-Heath CA, Sarnaik R, Wang BS, Liu X, Cang J (2009) Direction-specific disruption of subcortical visual behavior and receptive fields in mice lacking the beta2 subunit of nicotinic acetylcholine receptor. J Neurosci 29:12909-12918.

Wang L, Sarnaik R, Rangarajan K, Liu X, Cang J (2010) Visual receptive field properties of neurons in the superficial superior colliculus of the mouse. J Neurosci 30:16573-16584.

Yang L, Lee K, Villagracia J, Masmanidis SC (2020) Open source silicon microprobes for high throughput neural recording. J Neural Eng 17:016036.

Zhao X, Liu M, Cang J (2014) Visual cortex modulates the magnitude but not the selectivity of looming-evoked responses in the superior colliculus of awake mice. Neuron 84:202-213. 